\title{
BMJ Open Retrospective review of abdominal aortic aneurysm deaths in New Zealand: what proportion of deaths is potentially preventable by a screening programme in the contemporary setting?
}

Wing Cheuk Chan, ${ }^{\circledR}$ Dean Papaconstantinou, Doone Winnard, Gary Jackson

To cite: Chan WC,

Papaconstantinou D, Winnard D, et al. Retrospective review of abdominal aortic aneurysm deaths in New Zealand: what proportion of deaths is potentially preventable by a screening programme in the contemporary setting? BMJ Open 2019;9:e027291. doi:10.1136/ bmjopen-2018-027291

- Prepublication history and additional material for this paper are available online. To view these files, please visit the journal online (http://dx.doi. org/10.1136/bmjopen-2018027291).

Received 17 0ctober 2018 Revised 25 June 2019 Accepted 08 July 2019

D) Check for updates

(c) Author(s) (or their employer(s)) 2019. Re-use permitted under CC BY-NC. No commercial re-use. See rights and permissions. Published by BMJ.

Population Health, Counties Manukau District Health Board, Auckland, New Zealand

Correspondence to

Dr Wing Cheuk Chan;

wingcheuk.chan@cmdhb.org.nz

\section{ABSTRACT}

Objectives To describe the proportions of people dying from abdominal aortic aneurysm (AAA) who might have benefited from a formal screening programme for AAA. Design Retrospective cross-sectional review of deaths. Setting and study populations All AAA deaths registered in New Zealand from 2010 to 2014 in the absence of a national AAA screening programme.

Main outcome measures Known history of AAA prior to the acute event leading to AAA death, prognosis limiting comorbidities, history of prior abdominal imaging and a validated multimorbidity measure (M3-index scores). Results 1094 AAA deaths were registered in the 5 years between 2010 and 2014 in New Zealand. Prior to the acute AAA event resulting in death, $31.3 \%$ of the cohort had a known AAA diagnosis, and $10.9 \%$ had a previous AAA procedure. On average, the AAA diagnosis was known 3.7 years prior to death. At least $77 \%$ of the people dying from AAA also had one or more other prognosis limiting diagnosis. The hazard of 1-year mortality associated with the non-AAA related comorbidities for the AAA cohort aged 65 or above were 1.5-2.6 times higher than to the age matched general population based on M3-index scores. In 2014 , overall AAA deaths accounted for only $0.7 \%$ of total deaths, and $1.0 \%$ of deaths among men aged 65 or above in New Zealand. At most, $20 \%$ of people dying from AAA in New Zealand between 2010 and 2014 might have had the potential to derive full benefit from a screening programme. About $51 \%$ of cases would have derived no or very limited benefit from a screening programme.

Conclusion Falling AAA mortality, and high prevalence of competing comorbidities and/or prior AAA diagnosis and procedure raises the question about the likely value of a national AAA screening programme in a country such as New Zealand.

\section{INTRODUCTION}

There has been increasing scrutiny in reviewing the balance between benefits and harms of screening programme. Screening for abdominal aortic aneurysm (AAA) is a contested topic, ${ }^{12}$ and the approach to population level screening is highly variable between different
Strengths and limitations of this study

- A retrospective review of abdominal aortic aneurysm (AAA) deaths in a country is a key component in evaluating the potential impact of a screening programme.

- This study provides a systematic method of/for describing multimorbidities of people who died from AAA in New Zealand.

- The disease definitions of this study are based on the International Classification of Diseases 10th Revision which are widely used internationally.

- The record linkages were carried out consistently using national unique identifiers for each individual.

- The percentage of prior abdominal aorta imaging is likely to be significantly underestimated because imaging scans in the community are not captured in coding routinely.

countries internationally. ${ }^{3}$ There are well established national screening programme for AAA in the UK and Sweden. ${ }^{45}$ The US Preventive Services Task Force and the Canadian Task Force on Preventive Health Care recommend one-time screening with ultrasonography for AAA for men who have ever smoked (from ages 65 to 75 years and from 65 to 80 , respectively). ${ }^{6} 7$ On the other hand, many other high-income countries such as Australia and New Zealand have yet to establish a national screening programme. ${ }^{89}$

Many evaluations of AAA screening programme predominately focus on case detection and process measures without formally undertaking linkage study to examine the likely impact from the AAA screening programme on overall mortality at a population level in the contemporary setting. ${ }^{10} 11$ While rapidly falling AAA prevalence and mortality prior to commencement of AAA screening in UK were acknowledged 
as important considerations in regard to cost effectiveness of a AAA screening programme, ${ }^{12-14}$ there has been less scrutiny on whether the rapidly evolving epidemiology has been captured appropriately in cost effectiveness studies that inform policy and commentaries. ${ }^{15-17}$

This study aims to design a method to review AAA deaths, using national data from New Zealand as an example, that can be replicable internationally to provide information on the potential value of a formal national AAA screening programme.

\section{Study aims to quantify the following}

1. The proportion of total deaths caused by AAA in New Zealand, and England and Wales from publicly available national mortality data, including a subgroup of men aged 65 and over which is a target population for screening in some countries.

Out of the deaths caused by AAA in New Zealand.

2. The proportion of AAA deaths that are highly unlikely to be prevented by AAA screening programme (eg, AAA diagnosis is known before the index event of AAA death, people who had received a prior imaging of the abdomen, people who already had prior AAA surgery).

3. The prevalence of competing comorbidities:

a. The proportion if AAA deaths were prevented, healthy life years would not be substantially prolonged (ie, people who had other prognosis limiting comorbidities).

b. The proportion who had other comorbidities that would likely impact on survival and/or quality of life and/or peri-operative complexity and costs.

4. The proportion of people who may have significant life years potentially gained by a AAA screening programme.

\section{METHODS}

\section{Data sources}

The following routine administrative datasets were sourced from the Analytical Services team from the New Zealand Ministry of Health:

- National Mortality Collection.

- National Minimum Dataset (Inpatients).

- New Zealand Cancer Registry.

All health service users in New Zealand are assigned a National Health Index (NHI). In this study the administrative data listed above are linked by an encrypted form of NHI, anonymously, at an individual level.

Deaths caused by AAA are identified from the National Mortality collection in New Zealand by the following International Statistical Classification of Diseases and Related Health Problems Codes, Tenth Revision (International Classification of Diseases (ICD) 10 codes): (I71.3, I74.4, I71.5, I71.6, I71.8, I71.9) from 2010 to 2014. The AAA deaths are analysed by age bands, gender, ethnicity and NZDep2013 Index of Deprivation. NZDep2013 is a relative geographical measure of socioeconomic deprivation, based on eight dimensions of deprivation, including income, employment, education qualifications, home ownership, household living space, access to car and communications, and family structure. ${ }^{18}$

The prior clinical diagnoses and imaging studies are identified by the hospitalisation events recorded in the National Minimum Datasets, or in the New Zealand Cancer Registry.

Any acute AAA hospital event with a discharge date that is within 2 days of the date of death from AAA are not included in the analyses because the diagnoses and comorbidities could potentially be complications related to the acute AAA presentation resulting in death.

If there are any prior continuous events that ended with a discharge date within 2 days of date of death and the event is coded as acute ('AC'), and has any AAA diagnosis (regardless of ruptured or not) at the same event, that is, ICD 10 codes I71.3-I71.9, then the event is excluded from the morbidity and prior imaging analyses. If there are any prior continuous events that with a discharge date within 2 days of date of death, and the event is coded as elective or arranged hospital event ('WN' or 'AA'), and has an AAA ruptured diagnosis for that event (ie, I71.3, I71.5, I71.8), then these events are excluded from the morbidity and prior imaging analyses, otherwise these events are included as part of the comorbidities analyses and calculation.

Prior morbidities and imaging studies in the last 15 years were arranged into five categories: A to E, with 24 diagnostic or medical history groupings are described below (detailed definitions are listed in online supplementary information).

A: Groups already identified with no or very little benefit to accrue from a national AAA screening programme

1. AAA diagnosis is known prior to death event in the absence of a formal screening programme.

2. Previous abdominal aortic intervention or surgery.

3. Previous abdominal ultrasound, CT scan or MRI in hospital (and hence these people are less likely to benefit from one-time screening).

B: Diagnoses that are strongly associated with poor prognosislikely little benefit if any would accrue from screening

1. Palliative care codes.

2. Metastatic cancer (from either cancer registry or NMDS). ${ }^{19-21}$

3. Upper gastrointestinal cancers. ${ }^{19-21}$

4. Lung cancer including mesothelioma. ${ }^{19-21}$

5. Respiratory failure. ${ }^{1920}$

6. Heart failure (including cor-pulmonale and cardiomyopathy)..$^{19-21}$

7. Cirrhosis and gastro-oesophageal varices. ${ }^{19-21}$

C: Diagnoses that would reduce life span and/or increase perioperative and postoperative complexity and costs—reduced benefit from screening

1. Chronic obstructive pulmonary disease (COPD) and bronchiectasis. $^{19-21}$

2. Cardiovascular disease (other than AAA). ${ }^{19-21}$ 
3. Atrial fibrillation and flutter. ${ }^{19-21}$

4. Other cardiac arrhythmia associated with perioperative complexity. ${ }^{19} 20$

5. Other malignant cancer, not including metastatic, upper gastrointestinal and lung cancer. ${ }^{1920}$

6. Diabetes. ${ }^{19-21}$

7. Chronic kidney disease stage three or above including renal failure. ${ }^{19-21}$

8. Receiving dialysis. ${ }^{19-21}$

D: Diagnoses that would reduce life span and associated with substantial reduction in quality of life - reduced absolute quantity and quality of life benefit from screening

1. Dementia. ${ }^{21}$

2. Hemiplegia or paraplegia. $^{21}$

3. Hip fracture. ${ }^{22}$

\section{E: Other relevant factors to consider}

1. History of smoking.

2. Current smoker.

3. Prior hospitalisations in the last 15 years.

The proportions of people who might have comorbidities similar to the participants of the Endovascular Aneurysm Repair (EVAR)-2 trial are estimated by counting the number of people who had any of the following: chronic kidney disease stage three or above including renal failure, cardiac arrythmia, COPD, respiratory failure, heart failure or cardiovascular disease. ${ }^{23}$

\section{Multimorbidity scores}

The M3 index score is a validated multimorbidity index derived from using log hazard ratios for 1-year mortality modelled from 61 categories of chronic conditions based on hospital discharge diagnoses in the past 5 years. ${ }^{20}$ The validation study (involving more than 1 million cases) has demonstrated that M3 index provides better prediction of 1-year mortality compared with Charlson or Elixhauser comorbidity scores based on c-statistics and integrated discriminative improvement. ${ }^{20} \mathrm{M} 3$ index has a positive $\log$ HR assigned for each of the 61 categories of chronic condition. A M3 index score of zero means the person does not have any of the chronic conditions diagnosed in a publicly funded hospital New Zealand in the past 5 years. To ensure comparability, the M3 index score for each individual was calculated using the identical chronic condition definition and methods as per original study with two exceptions..$^{20}$ First, any AAA diagnoses are excluded from the M3 index score calculations. Second, the M3 index scores calculated for this study did not include any diagnoses from AAA hospital events that ended 2 days prior to death as described by the exclusion rules of comorbidities analyses method above. This step is to ensure any complications or diagnosis arise from the final event resulting in death are not inadvertently misclassified as known comorbidities prior to the final event resulting in death. ${ }^{20}$ This exclusion rule is expected to result in more conservative estimates of comorbidities, as some relevant diagnoses not related AAA rupture would also be excluded at the final event, for example, metastatic cancer. The more conservatively calculated M3 index scores of people who died from AAA were compared with the age specific scores of the general population in New Zealand. ${ }^{20}$

\section{Patient and public involvement}

This study was carried out based on anonymous administrative data with no active patient or public involvement.

\section{Ethical approval}

As all datasets were entirely based on anonymous administrative data with no active patient involvement, no formal ethical review was required as per New Zealand ethical guidelines (from New Zealand Health and Disability Ethics Committees). ${ }^{24}$

\section{RESULTS}

There were 1094 deaths caused by AAA registered in the 5 years between 2010 and 2014 in New Zealand. The majority of the AAA deaths $(77.5 \%, \mathrm{n}=848)$ were people aged 75 or above (table 1). Males aged 65 or above accounted for $54 \%(n=596)$ of total AAA deaths. In 2014, overall AAA deaths accounted for only $0.7 \%$ of total deaths, and $1.0 \%$ of deaths among men aged 65 or above in New Zealand. This compares to England and Wales in 2015, where AAA accounted for $1.1 \%$ of total deaths as shown in tables 1 and 2 of online supplementary information. ${ }^{25}$

Out the 1094 AAA deaths, 31.3\% had a known AAA diagnosis coded in a previous hospitalisation prior to the acute AAA event resulting in death (table 2). On average, the AAA diagnosis was known 3.7 years prior to death, with $58 \%$ of the AAA diagnosis known 2 years prior to death. Consistent with known AAA diagnosis, $32.9 \%(\mathrm{n}=360)$ had a prior ultrasound, CT or MRI scan of the abdomen in hospital before the final acute AAA event. Out of the people $(n=360)$ who had documented abdominal imaging, the first scans were carried out on average 4.2 years prior to death. Within the 15 years prior to death, 139 people had more than one scan (ie, had evidence of follow-up imaging studies). Many scans were relatively recent, with $43 \%$ of people having an imaging scan of the abdomen within the year prior to death and before the final acute AAA event. Moreover, $10.9 \%$ of the people dying from a AAA event had at least one previous abdominal aortic surgical intervention.

People who died from AAA often had competing comorbidities associated with a reduction in life span and/or quality of life. For example, $21.1 \%$ of people who died from AAA also had a heart failure hospitalisation prior to the acute event. Smoking related comorbidities were common: $52.9 \%$ had evidence of other cardiovascular disease other than AAA, 25.6\% ( $\mathrm{n}=280)$ had evidence of malignant cancer and $18.6 \%$ had COPD and/or bronchiectasis. Furthermore, $19.9 \%$ had dementia, hemiplegia, paraplegia and/or hip fracture. In fact, $77 \%$ of AAA 
Table 1 Demographic characteristics of the deaths caused by AAA in New Zealand 2010-2014

\begin{tabular}{lcc}
\hline Characteristics & $\begin{array}{l}\text { Number of AAA } \\
\text { deaths }\end{array}$ & AAA deaths (\%) \\
\hline Age bands (years) & & \\
$35-44$ & 1 & 0.1 \\
$45-54$ & 2 & 0.2 \\
$55-64$ & 43 & 3.9 \\
$65-74$ & 200 & 18.3 \\
$75-84$ & 432 & 39.5 \\
\hline 85 and over & 416 & 38.0 \\
\hline
\end{tabular}

\section{Gender}

\begin{tabular}{lcc|}
\hline Females & 466 & 43 \\
\hline Males & 628 & 57 \\
\hline Ethnicity & & 7.4 \\
\hline Māori & 81 & 2.0 \\
\hline Pacific & 22 & 1.7 \\
\hline Asian & 19 & 88.8 \\
\hline European/Other & 972 & \\
\hline NZDep2013 Index of Deprivation & 2 \\
\hline Unknown & 24 & 5 \\
\hline 1 (least deprived & 56 & \\
areas) & & 8 \\
\hline 2 & 87 & 7 \\
\hline 3 & 81 & 9 \\
\hline 4 & 94 & 9 \\
\hline 5 & 96 & 12 \\
\hline 6 & 131 & 14 \\
\hline 7 & 155 & 11 \\
\hline 8 & 123 & 11 \\
\hline 9 & 120 & 12 \\
\hline 10 (most deprived & 127 & \\
areas) & & \\
\hline Total & 1094 & \\
\hline AAA, & & \\
\hline
\end{tabular}

AAA, abdominal aortic aneurysm.

deaths had one or more of comorbidities as defined by this study (Groups 4-21 as shown in table 2).

Up to $64 \%(n=740)$ of all AAA deaths might have been potential candidates for the EVAR Trial 2 from a comorbidity point of view, ${ }^{23}$ namely people who had renal dysfunction, cardiac arrythmia, COPD, respiratory failure, heart failure and underlying cardiovascular disease.

Of those people who had a prior AAA diagnosis $(\mathrm{n}=342), 31 \%$ had received abdominal aortic surgery in the past. Overall, $32.4 \%(\mathrm{n}=354)$ either had a prior AAA diagnosis or received abdominal aortic surgery. Out of those people with prior AAA diagnosis and who had not had abdominal aortic surgery $(n=235), 46 \%, 89 \%$ and $26 \%$ had at least one comorbidity within categories B, C and $\mathrm{D}$ respectively. Excluding those who had either prior
AAA diagnosis, imaging or diagnoses strongly associated with poor prognosis (ie, those in categories A and B), this leaves $48.6 \%$ of the cohort $(n=532)$. Out of the 532 deaths, $58 \%(\mathrm{n}=309)$ had comorbidities (categories C and D) that would limit the benefits they would likely derive from a screening programme. In other words, $28 \%$ of overall AAA deaths $(n=309)$ might be expected to accrue only a partial benefit from the screening programme because of competing comorbidities (did not have category A or $\mathrm{B}$ but have $\mathrm{C}$ or $\mathrm{D}$ ). The subgroup analysis of men aged 65 or above, showed a very similar pattern of prior AAA diagnosis and comorbidities except for conditions that are known to have a major gender difference such as hip fracture. In summary, at most $20.4 \%$ of people dying from AAA in New Zealand might have the potential to accrue full benefit from a screening programme (people who did not have category A, B, C, or D). The overlap between categories is shown in online supplementary figure 1 of the online supplementary information.

People who died from AAA often had more than one prognosis-limiting disease category. More than $56 \%$ of AAA deaths had a prior hospitalisation with two or more diagnostic groups of comorbidities as defined by this study (within groups 3-21).

\section{M3 index score}

Consistent with the descriptive findings (tables 2 and 3), $67 \%(\mathrm{n}=738)$ of the AAA cohort had a M3 index score $>0$ (excluding AAA diagnosis from the M3 index scores) and had a much higher age specific M3 index score distribution compared with the general population in New Zealand (table 4).$^{20}$ Comparing the median M3 index scores for ages 65 or above, the hazard of 1-year mortality associated with the non-AAA related comorbidities for the AAA cohort was 1.5-2.6 times higher than compared with the age matched general population.

\section{DISCUSSION}

AAA incidence and mortality has fallen substantially in many developed countries since the recruitments for the four randomised controlled trials of AAA screening from 1988 to $1999 .{ }^{12}{ }^{26-31}$ Particularly in countries where AAAs account for decreasing proportions of overall deaths, this study suggests a retrospective review of recent AAA deaths could be a helpful approach to quantify the potential impact of a screening programme for AAA in the contemporary setting. One of the primary objectives for screening for AAA is to reduce all-cause mortality at a population level. The all-cause mortality benefit from the four published randomised control trials of AAA screening has been a subject of intense debate predominately on the basis of statistical significance and modelling methods. ${ }^{32-35}$ However, there is little discussion on whether the claimed $2 \%$ absolute all-cause mortality benefit demonstrated in trials at a time of higher incidence of AAA is still feasible in the context of falling AAA incidence and AAA mortality, considering AAA deaths only account for about $1 \%$ of 
Table 2 Known medical history and comorbidities of people who died from AAA in New Zealand from 2010 to 2014 prior to the acute AAA event

\begin{tabular}{|c|c|c|c|c|}
\hline Description & Number & $\begin{array}{l}\text { AAA deaths } \\
(\%)\end{array}$ & $\begin{array}{l}\text { Number (men } \\
\text { aged } \geq 65)\end{array}$ & $\begin{array}{l}\text { AAA deaths } \\
\text { (men aged } \\
\geq 65 \text { ) (\%) }\end{array}$ \\
\hline 1. Known AAA diagnosis & 342 & 31.3 & 186 & 31.2 \\
\hline 2. Previous abdominal aortic surgery & 119 & 10.9 & 69 & 11.6 \\
\hline 3. Previous abdominal CT/MRI scan & 360 & 32.9 & 190 & 31.9 \\
\hline $\begin{array}{l}\text { A. Unlikely to benefit from a national AAA screening programme } \\
\text { (groups 1-3) }\end{array}$ & 456 & 41.7 & 244 & 40.9 \\
\hline 4. Palliative code & 46 & 4.2 & 24 & 4 \\
\hline 5. Metastatic cancer & 24 & 2.2 & 12 & 2 \\
\hline 6. Upper gastrointestinal cancers & 2 & 0.2 & 1 & 0.2 \\
\hline 7. Lung cancer including mesothelioma & 15 & 1.4 & 11 & 1.8 \\
\hline 8. Respiratory failure & 35 & 3.2 & 23 & 3.9 \\
\hline 9. Heart failure (including cor-pulmonale) & 231 & 21.1 & 126 & 21.1 \\
\hline 10. Cirrhosis, gastro-oesophageal varices & 8 & 0.7 & 4 & 0.7 \\
\hline $\begin{array}{l}\text { B. Diagnoses that are strongly associated with poor prognosis } \\
\text { (groups } 4-10 \text { ) }\end{array}$ & 310 & 28.3 & 170 & 28.5 \\
\hline 11. COPD and bronchiectasis & 204 & 18.6 & 116 & 19.5 \\
\hline 12. Cardiovascular disease (excluding AAA) & 579 & 52.9 & 336 & 56.4 \\
\hline 13. Atrial fibrillation and flutter & 255 & 23.3 & 153 & 25.7 \\
\hline 14. Other cardiac arrhythmia & 93 & 8.5 & 54 & 9.1 \\
\hline $\begin{array}{l}\text { 15. Other malignant cancer, excluding metastatic, upper } \mathrm{Gl} \text { and } \\
\text { lung cancer }\end{array}$ & 268 & 24.5 & 165 & 27.7 \\
\hline 16. Diabetes & 109 & 10.0 & 65 & 10.9 \\
\hline 17. Chronic kidney disease stage 3 or above & 109 & 10.0 & 65 & 10.9 \\
\hline 18. Received dialysis & 11 & 1.0 & 7 & 1.2 \\
\hline $\begin{array}{l}\text { C. Diagnoses that reduce life span and/or increase operative } \\
\text { complexity and cost (groups 11-18) }\end{array}$ & 806 & 73.7 & 451 & 75.7 \\
\hline 19. Dementia & 67 & 6.1 & 31 & 5.2 \\
\hline 20. Hemiplegia or paraplegia & 100 & 9.1 & 54 & 9.1 \\
\hline 21. Hip fracture & 78 & 7.1 & 23 & 3.9 \\
\hline $\begin{array}{l}\text { D. Diagnoses that reduce life span and associated with } \\
\text { substantial reduction in quality of life (groups 19-21) }\end{array}$ & 218 & 19.9 & 99 & 16.6 \\
\hline Have category A or B & 562 & 51.4 & 301 & 50.5 \\
\hline Did not have Category A or B & 532 & 48.6 & 295 & 49.5 \\
\hline Did not have Category A or B but have C or D & 309 & 28 & 175 & 29.3 \\
\hline Have category B, C or D & 842 & 77.0 & 467 & 78.4 \\
\hline Category A, B, C or D & 871 & 79.6 & 476 & 79.9 \\
\hline Did not have Category A, B, C or D & 223 & 20.4 & 120 & 20.1 \\
\hline 22. History of smoking & 579 & 52.9 & 365 & 61.2 \\
\hline 23. Current smoker & 335 & 30.6 & 186 & 31.2 \\
\hline 24. Prior hospitalisations in the last 15 years & 997 & 91.1 & 536 & 89.9 \\
\hline Total deaths & 1094 & & 596 & \\
\hline
\end{tabular}

AAA, abdominal aortic aneurysm.

total deaths in many developed countries such as UK and USA. ${ }^{134} 35$ The systematic reviewers for the US Preventive Services Taskforce indicated that all-cause mortality benefit from AAA screening is not convincing because AAA deaths are more likely to occur at older age and people with AAA often have other competing causes of death. ${ }^{32}$ Furthermore, 
Table 3 Number of people who died from AAA by the number of diagnostic groups of comorbidities

\begin{tabular}{lcl}
$\begin{array}{l}\text { Number of diagnostic } \\
\text { groups of comorbidities }\end{array}$ & $\begin{array}{l}\text { Number of } \\
\text { people }\end{array}$ & $\begin{array}{l}\text { AAA deaths } \\
(\%)\end{array}$ \\
\hline 0 & 252 & 23.0 \\
1 & 224 & 20.5 \\
2 & 225 & 20.6 \\
\hline 3 & 175 & 16.0 \\
4 & 111 & 10.1 \\
\hline 5 & 65 & 5.9 \\
6 & 30 & 2.7 \\
7 & 10 & 0.9 \\
8 & 2 & 0.2 \\
Total & 1094 & \\
\hline
\end{tabular}

AAA, abdominal aortic aneurysm.

a recent matched cohort study suggested that the absolute benefit from AAA screening for men in Sweden was only $7 \%$ of the absolute benefit demonstrated by the Multicentre Aneurysm Screening Study (MASS) randomised trial at two avoided AAA deaths from 10000 invitees. ${ }^{36}$ This present study has further consolidated the evidence base that screening for AAA is likely to have very limited impact (if any) on all-cause mortality because of the below reasons.

Only small proportions of people dying from AAA in contemporary settings have the potential to receive the full benefit from a screening programme

In the absence of a formal AAA screening programme in New Zealand, this study demonstrated at most $20.4 \%$ of people dying from AAA in New Zealand might have the potential to derive full benefit from a screening programme. In addition, $28 \%$ of people might only receive a partial benefit from the screening programme because of competing comorbidities.

Relatively large proportion of AAA were already identified prior to the acute event leading to AAA deaths in the absence of a formal screening programme

The diagnosis of AAA was relatively high $(31.3 \%)$ prior to the acute AAA event resulting in death. The majority of prior AAA diagnoses were known more than 2 years prior to the AAA deaths. Consistent with the high level of prior diagnosis, $32.9 \%$ of people dying from AAA had at least one documented abdominal imaging prior to the acute AAA event resulting in death. Just under $11 \%$ had prior abdominal aortic intervention. The combination of these three groups would mean $41.7 \%$ of the AAA deaths would receive very minimal or no benefit from a one-off AAA screening. This study's findings are consistent with the claim made by a recent Sweden cohort study that a large proportion of AAA were already identified before rupture. ${ }^{36}$ Their study concluded that the lack of statistical reduction in AAA mortality attributed to a AAA screening programme in Sweden may be associated with the high level of background diagnosis of AAA. ${ }^{36}$ Indeed, the increase in opportunistic detection and diagnosis of AAA is likely to be associated with the rapid increase in the use of abdominal imaging since $1990 .{ }^{37}$

\section{People who die from AAA have a high prevalence of competing comorbidities}

Despite excluding the AAA diagnosis and some of the diagnoses made in hospital events proximal to death as part of the M3 index score, people who died from AAA had a much higher level of complexity of comorbidities than the age matched general population in New Zealand

Table 4 Comparison between age specific M3 index scores (log HRs) between people who died from AAA and the general population in New Zealand

\begin{tabular}{|c|c|c|c|c|c|c|c|c|}
\hline \multirow[b]{2}{*}{ Age } & \multirow[b]{2}{*}{$\begin{array}{l}\text { Number of } \\
\text { AAA deaths }\end{array}$} & \multicolumn{4}{|c|}{$\begin{array}{l}\text { M3 index scores of people who died from AAA prior } \\
\text { to acute AAA event }\end{array}$} & \multicolumn{3}{|c|}{ M3 index scores of general population } \\
\hline & & Average & Median & $\begin{array}{l}\text { 75th } \\
\text { percentile }\end{array}$ & $\begin{array}{l}\text { 90th } \\
\text { percentile }\end{array}$ & Median & $\begin{array}{l}\text { 75th } \\
\text { percentile }\end{array}$ & $\begin{array}{l}\text { 90th } \\
\text { percentile }\end{array}$ \\
\hline $35-39$ & 1 & 0.33 & 0.33 & 0.33 & 0.33 & 0 & 0 & 0 \\
\hline $40-44$ & 0 & - & - & - & - & - & - & - \\
\hline $50-54$ & 2 & 0.19 & 0.19 & 0.28 & 0.34 & 0.0 & 0.0 & 0.2 \\
\hline $55-59$ & 10 & 1.04 & 0.82 & 1.44 & 2.83 & 0.0 & 0.0 & 0.3 \\
\hline $60-64$ & 33 & 0.55 & 0.00 & 0.64 & 1.85 & 0.0 & 0.0 & 0.4 \\
\hline $65-69$ & 78 & 0.78 & 0.41 & 0.99 & 2.00 & 0.0 & 0.0 & 0.6 \\
\hline
\end{tabular}

AAA, abdominal aortic aneurysm. 
as demonstrated by the comparison of the M3 index scores. Since tobacco smoking is strongly associated with $\mathrm{AAA},{ }^{31}$ it was not surprising to find that people who died from AAA also had many competing comorbidities which would limit the quality and quantity of life. Indeed, the subgroup with known AAA who had not received abdominal aortic interventions more often had more comorbidities that limit life span such as metastatic cancer and heart failure. This association is likely to be a challenge for predictive models to stratify the population for those best suited for screening, as people with higher risk of AAA may also be of higher risk of multimorbidity limiting their benefit from screening. The high prevalence of competing morbidities demonstrated in this study may also explain why it is challenging for newer surgical techniques such as EVAR to improve long term survival of people with AAA beyond 1 year, even though EVAR has significantly improved 30-day mortality compared with open repair. ${ }^{38}$ Indeed, subgroup analyses from a recent individual level meta-analysis of randomised controlled trials comparing EVAR and open repair did not find early benefit from EVAR for people with moderate renal dysfunction or coronary heart disease. ${ }^{38}$ Nevertheless, it would be important for policy makers to consider any new evidence that becomes available in regard to the longer term outcomes of new technological advances.

While many of the morbidities examined by this study are not absolute contraindications to abdominal aortic intervention, they often increase perioperative complexity and overall intervention costs as well as being associated with increased risk of postoperative complications and adverse events. In the context of inevitably treating people with AAA of whom the AAA would have otherwise never caused harm in their life time, it is important to consider the decision to undertake AAA intervention does not always translate to meaningful benefit to patients overall in terms of quality and/or quantity of life.

The EVAR-2 trial demonstrated that for people with AAA $(\geq 5.5 \mathrm{~cm})$ who were not eligible for open repair because of comorbidities, EVAR reduced aneurysm related mortality without increasing overall survival. ${ }^{23} \mathrm{Up}$ to $64 \%(n=740)$ of people who died from AAA might have been potential candidates for the EVAR Trial $2,{ }^{23}$ namely people who had renal dysfunction, cardiac arrythmia, COPD, respiratory failure, heart failure and underlying cardiovascular disease. Preventing a person from AAA rupture alone without significantly prolonging quantity and quality of life because of other morbidities may not be considered as desirable from a people centric point of view. This study highlights the type of common comorbidities that may be worth reviewing and optimising clinically before aortic intervention, as well as allowing a more informed consent about the likely benefits and potential harms related to the aortic intervention. For example, having an accurate assessment of a cancer prognosis or the functional status of people with dementia as appropriate; or optimising cardiovascular disease and heart failure management prior to proposed interventions.
Opportunity to improve outcomes of people with AAA without implementing a AAA screening programme

Many of the wider potential benefits from a AAA screening programme could potentially be achieved without implementing a AAA screening programme. For example, better use of technology and quality assurance of vascular surgery can be carried out without the additional costs and harms related to a screening programme. The cohorts with known AAA or who have had prior AAA intervention are more likely to benefit from systematic quality improvement efforts towards surveillance and the management pathway. These quality improvement efforts can be implemented without a formal screening programme. The benefit of this approach is that it can limit the harms and overdiagnoses related to a screening programme which usually has a primary aim to detect AAA cases. ${ }^{10}$

For people with known AAA, the absolute risk of AAA rupture resulting in death is actually low. In people with AAA who would normally be entered into a surveillance programme $(3.0-5.4 \mathrm{~cm})$, rupture accounts for about $1 \%-1.6 \%$ of all deaths. ${ }^{39}$ In the NHS AAA screening programme, the rupture risk of the 13000 men under surveillance is reported to be $<0.5 \% 0 .{ }^{4}$ Even for people with $5.5-6.9 \mathrm{~cm}$ AAA which is surgically indicated for AAA repair, the risk of rupture is estimated to be less than 5\% per year. ${ }^{40}$ In the context of competing comorbidities, about half of the large AAA who did not have operations died from other causes. ${ }^{40}$ Therefore, there is time to optimise management of comorbidities before elective intervention, or conservative treatment may be appropriate in some cases.

In the contemporary setting, other potential health and cardiovascular benefits from AAA screening programme such as improving CVD management, and support for smoking cessation, are less likely to be realised in countries such as New Zealand where coverage of the eligible population for cardiovascular risk assessment is at $90 \%{ }^{41}$ Indeed, a weighted meta-regression study of 10 articles demonstrated that out of a group of people with AAA $(3.0-5.4 \mathrm{~cm})$, there were eight times more deaths from other cardiovascular causes than from AAA rupture, emphasising the importance of CVD management in people with AAA.$^{39}$ If one is concerned about CVD risk, then designing interventions to more directly improve systematic CVD risk management in primary care could be suggested without the additional cost and potential harms related to a AAA screening programme.

\section{Time to revise the inputs and assumptions of published cost} effectiveness studies that have informed policy

One has to be cautious in accepting the frequently quoted claims that AAA screening remains cost effective as long as AAA prevalence is above $0.35 \%$ based on one-way sensitivity analysis. ${ }^{416}$ Many assumptions of such cost effectiveness models deserve more scrutiny and re-calibration with contemporary data and latest evidence is urgently required to better inform policy. For example, the cost 
effectiveness model for the NHS screening programme is based on 10-year results from the MASS trial. ${ }^{16}$ The absolute benefit from the screening programme is expected to be much less than the results from the MASS trial in the context of rapidly falling AAA mortality with AAA accounting for proportionally less of total deaths. Indeed, contemporary real world impact observed may be substantially less than demonstrated by historical randomised controlled trials. ${ }^{36}$ The lack of statistically significant AAA mortality benefit in the Western Australia Trial may be at least partly related to the high background abdominal screening. ${ }^{49}$ The increase of prior diagnosis and background abdominal scans in the contemporary setting would also be expected to reduce the relative benefit of the screening programme. This study reports $32.4 \%$ of the AAA deaths $(n=354)$ either had a prior AAA diagnosis or received abdominal aortic surgery before the acute AAA event leading to death and $41.7 \%$ had prior CT/ MRI scan of the abdomen, or a AAA diagnosis or aortic procedure. This compares to a recent cost effectiveness model output of only $19 \%$ of AAA rupture incidentally detected prior to rupture. ${ }^{42}$

The UK cost effectiveness model would not be realistic in New Zealand setting as it assumed AAA accounted for $3.6 \%$ and $2.3 \%$ of total deaths in the controlled and invited group claiming an absolute benefit of $0.9 \%$ of overall mortality from screening. ${ }^{16}$

Consistent with published literature ${ }^{32}$ this study clearly demonstrates people who died from AAA had substantially more complex comorbidities associated with 1-year mortality compared with the general population as demonstrated by M3 index scores. Indeed, the median survival of trial participants from EVAR-1, Dream, OVER and ACE at an average age of 71.7 years was around 8-9 years. ${ }^{38}$ This was lower than the life expectancy of the general UK population at aged 72 which was 13.3 years for males and 15.3 years for females. ${ }^{43}$ However, many of the cost effectiveness models assume that people with AAA have same age specific mortality and quality of life as the age matched population. ${ }^{16} 1742$ This unrealistic assumption is likely to result in substantially over estimating the benefits in life years and Quality Adjusted Life Years.

Finally, all the relevant costs of the screening pathway are often not adequately captured by cost effectiveness modelling. For example, the perioperative and longterm complications from AAA repairs are not insignificant. Aneurysm-related intervention or intervention for complications at 8 years were estimated to be $25 \%$ for endovascular repair and $20.6 \%$ for open repair. ${ }^{44}$ While the latest cost effectiveness model for AAA screening has made some technical improvements in some parts of the modelling, complications rates are informed by the EVAR-1 trial only. ${ }^{42}$ The prognosis, complication rates and costs for people with comorbidities such as candidates of EVAR-2 trial are not adequately captured resting in a more optimistic result. ${ }^{23}$ Moreover, cost effective models for AAA screening do not have documented methods to account for the wider range of practical costs related to implementing the AAA screening pathway in the real world such as proportions of patients who did not attend screening or surgical appointments. ${ }^{16} 17$

\section{Strengths and limitations of the study}

This study is based on ICD coding of administrative data which is widely available internationally and is able to cover the whole country, limiting selection biases. The methods could be replicated in other countries to inform continual quality improvement of existing screening programme as well as investment and disinvestment decisions. Indeed, the findings of this study could be used to validate many of the inputs, outputs and assumptions of cost effectiveness models of AAA screening that inform policy and quality improvement efforts. ${ }^{16} 1742$ The choice of prognostic limiting diagnoses were informed by the review of the medical literature ${ }^{19}$ or based on disease categories that had empirical data to demonstrate the association of high short-term mortality risk, ${ }^{20} 22$ and/ or had been associated with high morbidity burden as defined by the disability weights from the Global Burden of Disease Study. ${ }^{21}$

This study has a number of limitations. The percentage of people who had prior abdominal aorta imaging is likely to be significantly underestimated because abdominal ultrasound scans are not routinely coded in the hospitalisation datasets, and outpatient, community or privately funded ultrasound, CT and MRI imaging of the abdominal aorta were not counted. The diagnoses examined were based on cancer registry or hospitalisation codes, but comorbidities diagnosed in the community or comorbidities yet to be diagnosed were not available to be examined. Furthermore, the definition of the disease groupings was designed to optimise specificity and clinical relevance to the AAA intervention pathway, and severe forms of the disease are chosen. For example, only a more severe forms of chronic kidney disease are examined. As a consequence, some disease definitions used by this study are different from the M3 index, although actual M3 index scores were also calculated using the official M3 index disease definition. These limitations mean this study will still tend to underestimate the impact of the factors examined on the estimation of benefits and harms of AAA screening for cost effectiveness modelling. The under capture of opportunistic imaging and comorbidities of this study means it would provide a relatively optimistic view of true screening benefits. However, we believe applying these assumptions will be more realistic for the contemporary situation than the assumptions being applied in the current cost effectiveness models. ${ }^{161742}$ It is possible that the number of AAA deaths might be under reported. However, the number of under reporting is likely to be very small if any because of several reasons. Imaging studies are widely accessible in an acute or elective setting, and publicly funded in New Zealand. Rupture of AAA is highly symptomatic, which may prompt the patient to seek care, or provide a co-lateral history that makes misclassification as a cause of death 
less likely. All sudden, unexpected or unexplained deaths are reported to the coroners in New Zealand. ${ }^{45}$ Additional investigations and autopsies are undertaken for cases where cause of death was uncertain and/or would be of public interest. Even if there are cases where the AAA rupture are misclassified, the deceased would have been likely to have significant comorbidities that would have significantly limited any potential benefit accrued from a screening programme. It is also possible the people with known AAA who died suddenly from other causes may be misclassified inappropriately as AAA. New Zealand Ministry of Health has reviewed mortality coding related to ruptured AAA and provided reassurance that there is no documented AAA death misclassified by coding $(\mathrm{C}$ Fowler, AAA deaths, Ministry of Health, personal communication, 2017).

Since most people with AAA died from competing comorbidities, ${ }^{39}$ a retrospective review of AAA deaths is less likely to find severe forms of comorbidities that are strongly life span limiting compared with a prospective study of people with known AAA at a time of death.

\section{CONCLUSION}

Falling AAA mortality, high level of background abdominal imaging and competing comorbidities are expected to substantially reduce the cost effectiveness of a screening programme for AAA. The data in our study suggests many published cost effectiveness modelling papers may over-estimate the potential benefit from formal screening. Reasons include falling AAA mortality, high level of comorbidities, future life expectancy post AAA repair and the impact of incidental detection not being sufficiently taken into account. The use of real-world data from the methods and results are likely to be helpful in calibrating those models to better inform prioritisation conversations with a variety of stakeholders, including community and patient groups, about a range of competing funding priorities.

Acknowledgements We would like to thank Roger Marshall for his review and comments of an earlier version of the manuscript and Mildred Lee for providing the images in scaled rectangle diagram. Special thanks to James Stanley for providing the age specific M3 index scores for the New Zealand general population in numerical forms that were published as a diagram in his published article.

Contributors WCC conceived and designed the study. WCC and DP sourced the data from the New Zealand Ministry of Health. DP and WCC undertook the data analyses. WCC drafted the initial manuscript. WCC, DP, DW, GJ contributed to the interpretations and discussion of results. WCC, DP, DW, GJ approved the final version of the article. WCC is the guarantor.

Funding The authors have not declared a specific grant for this research from any funding agency in the public, commercial or not-for-profit sectors.

Competing interests None declared.

Patient consent for publication Not required.

Provenance and peer review Not commissioned; externally peer reviewed.

Data availability statement The data set from this study is held securely in encrypted NHI in Counties Manukau District Health Board. The Memorandum of Understanding with New Zealand Ministry of Health does not allow data sharing with external parties.
Open access This is an open access article distributed in accordance with the Creative Commons Attribution Non Commercial (CC BY-NC 4.0) license, which permits others to distribute, remix, adapt, build upon this work non-commercially, and license their derivative works on different terms, provided the original work is properly cited, appropriate credit is given, any changes made indicated, and the use is non-commercial. See: http://creativecommons.org/licenses/by-nc/4.0/.

\section{REFERENCES}

1. Johansson M, Hansson A, Brodersen J. Estimating overdiagnosis in screening for abdominal aortic aneurysm: could a change in smoking habits and lowered aortic diameter tip the balance of screening towards harm? BMJ 2015;350:h825.

2. Acosta $S$. Screening men for AAA under magnification loupe in Sweden. Lancet 2018;391:2394-5.

3. Stather PW, Dattani N, Bown MJ, et al. International variations in AAA screening. Eur J Vasc Endovasc Surg 2013;45:231-4.

4. Earnshaw JJ, Lees T. Update on screening for abdominal aortic aneurysm. Eur J Vasc Endovasc Surg 2017;54:1-2.

5. Wanhainen A, Hultgren R, Linné A, et al. Outcome of the Swedish nationwide abdominal aortic aneurysm screening program. Circulation 2016;134:1141-8.

6. LeFevre ML, U.S. Preventive Services Task Force. Screening for abdominal aortic aneurysm: U.S. preventive services Task force recommendation statement. Ann Intern Med 2014;161:281-90.

7. Canadian Task Force on Preventive Health Care. Recommendations on screening for abdominal aortic aneurysm in primary care. CMAJ 2017;189:E1137-E1145.

8. Robinson D, Mees B, Verhagen $\mathrm{H}$, et al. Aortic aneurysms - screening, surveillance and referral. Aust Fam Physician 2013;42:364-9.

9. Nair N, Sarfati D, Shaw C. Population screening for abdominal aortic aneurysm: evaluating the evidence against screening criteria. $N Z$ Med J 2012;125:72-83.

10. Jacomelli J, Summers L, Stevenson A, et al. Impact of the first 5 years of a national abdominal aortic aneurysm screening programme. Br J Surg 2016;103:1125-31.

11. Jacomelli J, Summers L, Stevenson A, et al. Editor's Choice Inequalities in Abdominal Aortic Aneurysm Screening in England: Effects of Social Deprivation and Ethnicity. Eur J Vasc Endovasc Surg 2017;53:837-43.

12. Choke E, Vijaynagar B, Thompson J, et al. Changing epidemiology of abdominal aortic aneurysms in England and Wales: older and more benign? Circulation 2012;125:1617-25.

13. Earnshaw JJ. Final say on research into population screening for abdominal aortic aneurysm. Angiology 2018;69:365-6.

14. Jacomelli J, Summers L, Stevenson A, et al. Update on the prevention of death from ruptured abdominal aortic aneurysm. J Med Screen 2017;24:166-8.

15. Kim LG, Thompson SG, Briggs AH, et al. How cost-effective is screening for abdominal aortic aneurysms? J Med Screen 2007; 14:46-52.

16. Glover MJ, Kim LG, Sweeting MJ, et al. Cost-Effectiveness of the National health service abdominal aortic aneurysm screening programme in England. Br J Surg 2014;101:976-82.

17. Svensjö S, Mani K, Björck M, et al. Screening for abdominal aortic aneurysm in 65-year-old men remains cost-effective with contemporary epidemiology and management. Eur J Vasc Endovasc Surg 2014;47:357-65.

18. Atkinson J, Salmond C, Crampton P. NZDep2013 index of deprivation. Wellington: University of Otago, 2014.

19. Jameson JL. Harrison's principles of internal medicine. 20th ed. New York: McGraw-Hill Education, 2018.

20. Stanley J, Sarfati D. The new measuring multimorbidity index predicted mortality better than Charlson and Elixhauser indices among the general population. J Clin Epidemiol 2017;92:99-110.

21. Global Burden of Disease Collaborative Network. Global burden of disease study 2016 (GBD 2016) disability weights. Seattle, United States: Institute for Health Metrics and Evaluation (IHME), 2017.

22. Bentler SE, Liu L, Obrizan M, et al. The aftermath of hip fracture: discharge placement, functional status change, and mortality. Am J Epidemiol 2009;170:1290-9.

23. Sweeting MJ, Patel R, Powell JT, et al. Endovascular repair of abdominal aortic aneurysm in patients physically ineligible for open repair: very long-term follow-up in the EVAR-2 randomized controlled trial. Ann Surg 2017;266:713-9.

24. National Ethics Advisory Committee. Ethical guidelines for observational studies: observational research, audits and related activities: revised edition. Wellington: Ministry of Health, 2012. 
25. Office for National Statistics. The 21st century mortality files population dataset, England and Wales, 2017. Available: https:// www.ons.gov.uk/peoplepopulationandcommunity/populationandmig ration/populationestimates/datasets/the21stcenturymortalityfiles populationdataset [Accessed 28 May 2018].

26. Thompson SG, Ashton HA, Gao L, et al. Final follow-up of the multicentre aneurysm screening study (mass) randomized trial of abdominal aortic aneurysm screening. Br J Surg 2012;99:1649-56.

27. Ashton HA, Gao L, Kim LG, et al. Fifteen-year follow-up of a randomized clinical trial of ultrasonographic screening for abdominal aortic aneurysms. Br J Surg 2007;94:696-701.

28. Lindholt JS, Sørensen J, Søgaard R, et al. Long-Term benefit and cost-effectiveness analysis of screening for abdominal aortic aneurysms from a randomized controlled trial. $\mathrm{Br} J$ Surg 2010;97:826-34.

29. McCaul KA, Lawrence-Brown M, Dickinson JA, et al. Long-Term outcomes of the Western Australian trial of screening for abdominal aortic aneurysms: secondary analysis of a randomized clinical trial. JAMA Intern Med 2016;176:1761-7.

30. Anjum A, Powell JT. Is the incidence of abdominal aortic aneurysm declining in the 21st century? Mortality and hospital admissions for England \& Wales and Scotland. Eur J Vasc Endovasc Surg 2012;43:161-6.

31. Sidloff D, Stather P, Dattani N, et al. Aneurysm global epidemiology study: public health measures can further reduce abdominal aortic aneurysm mortality. Circulation 2014;129:747-53.

32. Guirguis-Blake JM, Beil TL, Senger CA, et al. Ultrasonography screening for abdominal aortic aneurysms: a systematic evidence review for the U.S. preventive services Task force. Ann Intern Med 2014;160:321-9.

33. Guirguis-Blake JM, Beil TL, Whitlock EP. Correction: ultrasonography screening for abdominal aortic aneurysms. Ann Intern Med 2016;164:70-2.

34. Lederle FA. Does abdominal aortic aneurysm screening save lives? JAMA Surg 2016;151:697-8.
35. Takagi $\mathrm{H}$, Ando $\mathrm{T}$, Umemoto $\mathrm{T}$, et al. Abdominal aortic aneurysm screening reduces all-cause mortality: make screening great again. Angiology 2018;69:205-11.

36. Johansson M, Zahl PH, Siersma V, et al. Benefits and harms of screening men for abdominal aortic aneurysm in Sweden: a registrybased cohort study. Lancet 2018;391:2441-7.

37. Raja AS, Mortele KJ, Hanson R, et al. Abdominal imaging utilization in the emergency department: trends over two decades. Int J Emerg Med 2011;4:19.

38. Powell JT, Sweeting MJ, Ulug P, et al. Meta-Analysis of individualpatient data from EVAR-1, DREAM, over and ACE trials comparing outcomes of endovascular or open repair for abdominal aortic aneurysm over 5 years. Br J Surg 2017;104:166-78.

39. Bath MF, Gokani VJ, Sidloff DA, et al. Systematic review of cardiovascular disease and cardiovascular death in patients with a small abdominal aortic aneurysm. Br J Surg 2015;102:866-72.

40. Scott SWM, Batchelder AJ, Kirkbride D, et al. Late survival in Nonoperated patients with infrarenal abdominal aortic aneurysm. Eur $J$ Vasc Endovasc Surg 2016;52:444-9.

41. Ministry of Health. How is my dhb performing? 2015/16 quarter three (January-March 2016) results, 2016. Available: http://www.health. govt.nz/system/files/documents/pages/health_target_quarter_three_ 2015-16 results for web.pdf [Accessed 11 Jul 2016].

42. Sweeting MJ, Masconi KL, Jones E, et al. Analysis of clinical benefit, harms, and cost-effectiveness of screening women for abdominal aortic aneurysm. Lancet 2018;392:487-95.

43. Office for National Statistics. National life tables: United Kingdom, 2017. Available: https://www.ons.gov.uk/peoplepopulationandc ommunity/birthsdeathsandmarriages/lifeexpectancies/datasets/nati onallifetablesunitedkingdomreferencetables [Accessed 16 Aug 2018].

44. Schermerhorn ML, Buck DB, O'Malley AJ, et al. Long-Term outcomes of abdominal aortic aneurysm in the Medicare population. N Engl J Med 2015;373:328-38.

45. Ministry of Justice. Annual report 2017/18. office of the chief coroner of new Zealand. Wellington Ministry of Justice; 2018. 